\title{
Nanoperforations in Poly(lactic acid)
}

\section{Free-Standing Nanomembranes to Promote}

\section{Interactions with Cell Filopodia}

Anna Puiggalí-Jou, ${ }^{1,2}$ Judith Medina, ${ }^{1,2}$ Luis J. del Valle ${ }^{1,2, *}$ and Carlos

\author{
Alemán ${ }^{1,2, *}$ \\ ${ }^{1}$ Departament d'Enginyeria Química, E. T. S. d'Enginyeria Industrial de Barcelona, \\ Universitat Politècnica de Catalunya, Diagonal 647, Barcelona E-08028, Spain \\ ${ }^{2}$ Centre for Research in Nano-Engineering, Universitat Politècnica de Catalunya, \\ Edifici C', C/Pasqual i Vila s/n, Barcelona E-08028, Spain
}

*luis.javier.del.valle@upc.edu and carlos.aleman@upc.edu 


\begin{abstract}
Nanoperforated poly(lactic acid) (PLA) free-standing nanomembranes (FsNMs) have been prepared using a two-step process: (1) spin-coating a mixture of immiscible polymers to provoke phase segregation and formation of appropriated nanofeatures (i.e. phase separation domains with dimensions similar to the entire film thickness); and (2) selective solvent etching to transform such nanofeatures into nanoperforations. For this purpose, PLA has been mixed with polyethylene glycol (PEG) and poly(vinyl alcohol) (PVA). Unfortunately, the characteristic of PLA:PEG mixtures were not appropriated to prepare nanoperforated FsNMs. In contrast, perforated PLA FsNMs with pores crossing the entire film thickness, which have been characterized by scanning electron microscopy and atomic force microscopy, were obtained using PLA:PVA mixtures. The diameter $(\phi)$ of such pores has been controlled through both the PLA:PVA ratio and the processing conditions of the mixtures, FsNMs with pores of $\phi \approx 0.8 \mu \mathrm{m}, 170 \mathrm{~nm}$ and 65 $\mathrm{nm}$ being achieved. Investigations on nanoperforated FsNMs (i.e. those with $\phi \approx 170$ and $65 \mathrm{~nm}$ ), which are the more regular, reveal that pores crossing the entire membrane thickness do not affect the surface wettability of PLA but drastically enhances the cellular response of this biomaterial. Thus, cell proliferation assays indicate that cell viability in PLA with perforations of $\phi \approx 170 \mathrm{~nm}$ is $\sim 2.6$ and $\sim 2.2$ higher than in nonperforated PLA and PLA with perforations of $\phi \approx 65 \mathrm{~nm}$, respectively. This excellent response has been attributed to the similarity between the nanoperforations with $\phi \approx 170$ $\mathrm{nm}$ and the filopodia filaments in cells $(\phi \approx 100-200 \mathrm{~nm})$, which play a crucial role in cell migration processes. The favorable interaction between the perforated membrane nanofeatures and cell filopodia has been corroborated by optical and scanning electron microscopies.
\end{abstract}


Keywords: Nanofeatures; Perforated nanomembranes; Phase segregation; Selfsupported nanomembranes; Tissue Engineering; Ultra-thin films; 


\section{INTRODUCTION}

The term giant nanomembrane was coined by Kunitake et al. [1] to denote selfsupporting membranes with thickness $(L)$ from 1 to $100 \mathrm{~nm}$ and an aspect ratio of size and thickness higher than $10^{6}$. Besides such characteristics, these free-standing nanomembranes (FsNMs) exhibit other special properties, such as easiness of handling, low weight, high flexibility, robustness and, in some cases, transparency [2]. In the last decade, polymeric FsNM, have emerged as versatile elements for biomedical applications as varied as overlapping therapy, burn wound infection treatment, antimicrobial platforms, scaffolds for tissue engineering, drug-loading and delivery systems, biosensors, etc., as recently reviewed Pérez-Madrigal et. al [3].

Among the synthetic polymers used to prepare FsNMs for biomedical applications, linear aliphatic polyesters, such as poly(lactic acid) (PLA) and poly(tetramethylene succinate) (PE44), have been extensively chosen since their biodegradation rate and mechanical properties can be easily controlled through variations in their molecular weight [3-7]. Within the specific case of PLA, Takeoka and co-workers [8] developed FsNMs with $L=23 \pm 5 \mathrm{~nm}$ by spin-coating polymer solutions onto poly(vinyl alcohol) (PVA) sacrificial films. The mechanical properties and adhesion strength exhibited by such PLA nanosheets, encouraged the analysis of their feasibility as a wound dressing [8,9]. On the other hand, Pensabene et al. [10] studied the biocompatibility, adhesion and proliferation activity of several cell types onto PLA FsNMs with an average thickness of $L=320 \pm 27 \mathrm{~nm}$, which were also prepared by spin-coating. Both, immortalized cell lines and primary cell lines cultured on those FsNMs exhibited good morphological and metabolic features and the ability to fully differentiate. Moreover, the effect of an underlying substrate on the interaction between cells and PLA FsNMs was recently investigated by collecting spin-coated nanosheets on both a stainless steel 
mesh and a $\mathrm{SiO}_{2}$ substrate [5]. Although topological and mechanical properties of PLA FsNMs did not influence cell viability after $24 \mathrm{~h}$ of culturing, cells did geometrically sense the stiffness of the underlying material, thus affecting the adhesion geometry.

The aim of this work is to improve the applicability of PLA FsNMs as flexible bioactive substrates for cell culture by introducing uniformly distributed nanoperforations in a controllable manner. More specifically, cell adhesion and spreading have been significantly enhanced by adapting the dimensions of nanopores, which might penetrate the entire thickness of the ultra-thin film, to those of cell nanofeatures (i.e. promoting cell-substrate interactions). For this purpose, nanoperforated FsNMs have been prepared by spin-coating mixtures of PLA and a polymer with poor affinity towards such polyester, to develop phase-segregated ultrathin films with a thickness comparable to the dimensional scale of the phase separation domains. Application of the selective solvent etching technique, which was introduced by Walheim et al. [11] to illustrate the influence of the relative solubility of polymer blends on the structure of the films, to the biphasic nanosheets has resulted in the formation of perforated PLA FsNMs. The combination of spin-coating and selective solvent etching was recently employed by Zhang and Takeoka [12] to study the phaseseparation mechanism of immiscible polystyrene (PS) : poly(methyl methacrylate) (PMMA) mixtures and to prepare nanoperforated PMMA ultra-thin films.

In this work, phase-segregated ultra-thin films have been prepared by combining PLA with polyethylene glycol (PEG) and PVA. However, surface nanofeatures compatible with the fabrication of nanoperforated FsNMs via selective solvent etching were only detected in PLA-PVA ultra-thin films. Cell viability assays using nonperforated and perforated PLA FsNMs have proved that the behavior of the latter as 
bioactive substrate is much better than that of the former, even though it drastically depends on the nanopore dimensions.

\section{METHODS}

\section{Materials}

PLA, a product of Natureworks (polymer 2002D), was kindly supplied by Nupik International (Polinyà, Spain). According to the manufacturer, this PLA has a D content of $4.25 \%$, a residual monomer content of $0.3 \%$, density of $1.24 \mathrm{~g} / \mathrm{cc}$, glass transition temperature $\left(T_{g}\right)$ of $58{ }^{\circ} \mathrm{C}$ and melting point of $153{ }^{\circ} \mathrm{C}$. The number and weight average molecular weights and polydispersity index, as determined by GPC, were $M_{n}=98,100$ $\mathrm{g} / \mathrm{mol}, M_{w}=181,000 \mathrm{~g} / \mathrm{mol}$ and 1.85 , respectively. Polyethylene glycol (PEG) with $M_{w}=$ $35,000 \mathrm{~g} / \mathrm{mol}$ was purchased from Sigma-Aldrich (Germany). PVA (87-89\% hydrolysed) with $M_{w}=13,000-23,000 \mathrm{~g} / \mathrm{mol}$ was purchased from Sigma-Aldrich (USA). Dry trichloromethane stabilized with 50 ppm of amylene DS-ACS (99.9\%) was purchased from Panreac Quimica S.A.U. (Spain). 1,1,1,3,3,3-Hexafluoroisopropanol (HFIP) was purchased from Apollo Scientific Limit (UK). $\mathrm{SiO}_{2}$ cover-glasses of $14 \mathrm{~mm}$ of diameter were acquired to Agar Scientific (France) while the glass cover slips, $22 \times 22$ $\mathrm{mm}^{2}$ were purchased at Menzel-Glässer (Germany).

For cell culture experiments, Madin-Darby Canine Kidney (MDCK) and African green monkey kidney (Vero) cells were purchased from ATCC, USA. Dulbecco's phosphate buffered saline (PBS) solution without calcium chloride and magnesium chloride, Dulbecco's modified Eagle's medium (DMEM, with $4500 \mathrm{mg} / \mathrm{L}$ of glucose, $110 \mathrm{mg} / \mathrm{L}$ of sodium pyruvate and $2 \mathrm{mM}$ of L-glutamine), penicillin-streptomycin, 3(4,5-dimethylthiazol-2-yl) 2,5-diphenyltetrazolium bromide (MTT, 97.5\%) and trypsin EDTA solution (0.05\% trypsin, $0.02 \%$ EDTA) were all purchased from Sigma-Aldrich, 
(USA). Fetal bovine serum (FBS) and trypan blue stain $(0.4 \%)$ were purchased from Gibco, UK.

\section{Preparation of solutions and mixtures}

For the fabrication of non-perforated PLA FsNMs, 50, 25 and $10 \mathrm{mg} / \mathrm{mL}$ polymer solutions in chloroform were prepared. Perforated FsNMs were obtained by blending PLA and PEG or PVA. PLA-PEG blends with 50:50, 60:40, 80:20 and 90:10 PLA:PEG $\mathrm{v} / \mathrm{v}$ ratios were prepared by combining PLA $(10 \mathrm{mg} / \mathrm{mL})$ and PEG $(10 \mathrm{mg} / \mathrm{mL})$ chloroform solutions. On the other hand, PLA-PVA blends with 80:20 and 90:10 PLA:PVA v/v ratios were obtained by mixing PLA $(10 \mathrm{mg} / \mathrm{mL})$ and PVA $(10 \mathrm{mg} / \mathrm{mL})$ HFIP solutions.

\section{Preparation of free standing nanomembranes}

FsNMs were prepared by applying the procedure described by Kunitake and coworkers [13], which was successfully used in previous works [6,7,14]. Firstly, a PVA solution in milliQ water $(100 \mathrm{mg} / \mathrm{mL})$ was spin-coated onto a glass slip, cleaned by successive sonication in acetone, ethanol, and water, at $2500 \mathrm{rpm}$ for $60 \mathrm{~s}$ to obtain a sacrificial layer. After this, PLA, PLA:PEG and PLA:PVA solutions were spin-coated at speeds ranging from 2500 to $7000 \mathrm{rpm}$ for $1 \mathrm{~min}$ to obtain ultra-thin films onto the sacrificial layer. Finally, samples were immersed into milliQ water for the separation of the nanomembrane from the substrate (i.e. dissolution of the sacrificial layer) as well as for the creation of pores (i.e. dissolution of PEG and PVA phases in PLA-PEG and PLA-PVA nanomembranes).

\section{Profilometry}


Film thickness measurements were carried out using a Dektak 150 stylus profilometer (Veeco, Plainview, NY). Different scratches were intentionally provoked on the nanomembranes and measured to allow statistical analysis of data. At least eighteen independent measurements were performed for two samples of each examined condition. Imaging of the films was conducted using the following optimized settings: tip radius $=65.5 \mu \mathrm{m}$; stylus force $=3.0 \mathrm{mg}$; scan length $=7 \mathrm{~mm}$; and speed $=100 \mu \mathrm{m} / \mathrm{s}$.

\section{Atomic force microscopy (AFM)}

The surface morphology and topography of nanomembranes supported onto glass slips, before and after application of selective solvent etching, were studied by AFM. Images were obtained with a Molecular Imaging PicoSPM using a NanoScope IV controller under ambient conditions. The tapping mode AFM was operated at constant deflection (i.e. vertical constant force with triangular shaped gold-coated silicon nitride). The row scanning frequency was set to 0.87 or $0.68 \mathrm{~Hz}$, depending on the sample response, and the physical tip sample motion speed was $10 \mathrm{~mm} / \mathrm{s}$.

RMS roughness $(\mathrm{Rq})$ and profile sections of the images were determined using the statistics application and tools of the NanoScope Analysis software version 1.20 (Bruker), which calculates the average considering all the values recorded in the topographic image with exception of the maximum and the minimum. The scan window sizes were $10 \times 10$ or $2 \times 2 \mu \mathrm{m}^{2}$.

\section{Scanning electron microscopy (SEM)}

Detailed inspection of nanomembranes before and after application of selective solvent etching was conducted by scanning electron microscopy. A Focus Ion Beam Zeiss Neon 40 instrument (Carl Zeiss, Germany) equipped with an energy dispersive X- 
ray (EDX) spectroscopy system and operating at 1 and $5 \mathrm{kV}$ for characterization of systems without and with cells, was used. Nanomembranes supported onto glass slips were mounted on a double-sided adhesive carbon disc. FsNMs were, firstly, mounted using silver staining and, after this, were sputter-coated with an ultra-thin carbon layer $(6-10 \mathrm{~nm})$ to prevent sample charging problems. The diameter of the perforations was measured with the SmartTiff software from Carl Zeiss SMT Ltd.

\section{Wettability}

Static contact angle measurements with the sessile drop method were recorded and analysed at room temperature on an OCA-15EC contact angle meter from DataPhysics Instruments $\mathrm{GmbH}$ with SCA20 software (version 4.3.12 build 1037). The solvents, which were used as received, considered for this study were:

- MilliQ water;

- $\quad N, N$-dimethyl formamide (DMF; Panreac, ultra-high purity);

- Ethylene glycol (EG; Merck >99.5\%);

The sessile drop was gently put on the surface of non-perforated and nanoperforated PLA using a micrometric syringe with a proper metallic needle (Hamilton $500 \mu \mathrm{L}$ ). The ellipse method was used to fit a mathematical function to the measured drop contour. For each solvent and PLA system, 20 drops were examined.

\section{Cell adhesion and cell proliferation assays}

Vero (African green monkey kidney epithelial-like cell line) and MDCK (Madin Darby canine kidney epithelial-like cell line) cells were cultured in DMEM supplemented with $10 \% \mathrm{FBS}, 1 \%$ penicillin and $1 \%$ streptomycin at $37^{\circ} \mathrm{C}$ in a 
humidified atmosphere of $5 \% \mathrm{CO}_{2}$ in air. Culture media were changed every three days. For sub-culture, cell monolayers were rinsed with PBS and detached by incubating them with $0.25 \%$ trypsin/EDTA for $5 \mathrm{~min}$ at $37^{\circ} \mathrm{C}$. Finally, cells were resuspended in $5 \mathrm{~mL}$ of fresh medium and their concentration was determined by counting at the Neubauer camera using $0.4 \%$ trypan blue as dye vital.

Perforated PLA nanomembranes were prepared by spin-coating on glass cover slips of $14 \mathrm{~mm}$ of diameter. Samples were placed in plates of 24 wells and sterilized using UV light for $15 \mathrm{~min}$ in a laminar flux cabinet. Controls were simultaneously performed by culturing cells on the surfaces of $\mathrm{SiO}_{2}$ wafers and non-perforated PLA nanomembranes. For adhesion assays, an aliquot of $1 \mathrm{~mL}$ containing $6 \times 10^{4}$ cells was deposited on the nanomembrane of each well. After $24 \mathrm{~h}$ non-attached cells were washed out while attached cells were quantified. For proliferations assays, the aliquot deposited on each well contained $4 \times 10^{4}$ cells. Quantification of proliferated cells was performed after 7 days of culture.

The percentage of cells adhered and proliferated was calculated through the MTT [3(4,5-dimethylthiazol-2-yl)-2,5-diphenyltetrazolium bromide] assay. This determines the cell viability [15]. Results were normalized to the non-perforated PLA control and represented as relative percentages. Specifically, $50 \mu \mathrm{L}$ of MTT solution $(5 \mathrm{mg} / \mathrm{mL}$ in PBS) were added to each well. After $2 \mathrm{~h}$ of incubation, samples were washed twice with PBS and stored in clean wells. In order to dissolve formazan crystals, $200 \mu \mathrm{L}$ of DMSO/methanol/water (70/20/10\% v/v) were added. Finally, the absorbance was measured at $570 \mathrm{~nm}$ through an ELISA reader (Biochrom, UK). Results were derived from the average of four replicates $(n=4)$.

After culture, samples were fixed in a $2.5 \%$ formaldehyde PBS solution overnight at $4{ }^{\circ} \mathrm{C}$. Then, they were dehydrated by washing in an alcohol battery $\left(30^{\circ}, 40^{\circ}, 70^{\circ}, 95^{\circ}\right.$ 
and $100^{\circ}$ ) at $4{ }^{\circ} \mathrm{C}$ for 15 min per wash. Finally, samples were air-dried and sputtercoated with carbon before SEM observation.

\section{RESULTS AND DISCUSSION}

\section{Non-perforated PLA free standing nanomembranes}

PLA FsNMs have been extensively studied [3,5-10] and, therefore, the aim of this sub-section is only to establish the influence of spin-coater controllable parameter in their most relevant nanofeatures. This information is relevant for the choice of the requirements subsequently employed in the preparation of perforated nanofilms.

In order to adjust the thickness, different PLA solutions in chloroform were spincoated onto glass slides for 1 min using different conditions. Table S1 indicates that the thickness of the films, as determined by contact profilometry, is severely affected by both the angular speed and the polymer concentration in the solution. Thus, although the variation of the thickness is inversely proportional to the speed in all cases, this phenomenon is more pronounced for the higher PLA concentrations, especially for the $50 \mathrm{mg} / \mathrm{mL}$ PLA solution. Among the tested conditions, membranes of nanometric thickness were only obtained with the lowest polymer concentration $(10 \mathrm{mg} / \mathrm{mL})$ and the highest spin-coater speed $(7000 \mathrm{rpm})$. Figure 1a evidences that PLA nanomembranes prepared under such conditions are very homogeneous and smooth, the roughness being of only $\mathrm{Rq}=1.1 \pm 0.2 \mathrm{~nm}$.

After this, PLA ultra-thin films were deposited onto a PVA sacrificial layer of $L=$ $238 \pm 43 \mathrm{~nm}$ and $\mathrm{Rq}=2.6 \pm 0.8 \mathrm{~nm}$ using the above selected conditions. After coating, samples were immersed into water for the separation of the PLA membrane. Profilometry and AFM measures indicated that both the thickness $(L=238 \pm 43 \mathrm{~nm})$ and roughness $(\mathrm{Rq}=2.6 \pm 0.8)$ increased significantly with respect to the membranes 
deposited onto glass slides. Thus, spreading of PLA onto PVA was worse than onto glass, which has been attributed to the fact that PLA...PVA interactions are more attractive than PLA $\cdots$ glass interactions.

\section{PLA-PEG nanomembranes}

Fabrication of nanoperforated FsNMs using a technique based on the phasesegregation of two polymers and subsequent elimination of one of them via selective solvent etching requires fulfilment of the following conditions:

- The two polymers must be soluble in a common solvent to facilitate the spincoating process.

- The two polymers must be immiscible to promote the phase separation.

- The two polymers should have similar molecular weights to facilitate the formation of nanofeatures (i.e. nanopores).

- The polymer used to fabricate the nanomembrane must remain unaltered by the solvent employed to dissolve the less abundant polymer.

In this work, the first attempt to obtain nanoperforated FsNMs was done using PLA and PEG. Both materials are soluble in chloroform, while PLA and PEG are insoluble and soluble in water, respectively. PLA-PEG nanomembranes were prepared by spincoating 50:50, 60:40, 80:20 and 90:10 PLA:PEG mixtures as described in the Methods section, and applying $7000 \mathrm{rpm}$ for $1 \mathrm{~min}$. Table 1 lists the thickness and roughness of the resulting nanomembranes, which are compared with those achieved for PLA under the same conditions, while the corresponding AFM images are displayed in Figure S1.

Although the incorporation of PEG affects significantly to both the thickness and roughness of PLA nanomembranes, these variations are apparently independent on the 
PEG concentration. Furthermore, no particular nanofeature is detected in the surface topography of 50:50, 80:20 and 90:10 PLA-PEG nanofilms, even though small shallow cavities are detected at the 60:40 membrane. The characteristics of PLA-PEG ultra-thin films are not consistent with a phase separation process and can be attributed to different reasons, as for example: (1) the evaporation rate of chloroform is not appropriated to produce instabilities at the polymer-polymer interface [16]; (2) the difference between the molecular weights of PLA and PEG is too large for the formation of PEG-like pores in the PLA matrix [17]; and (3) the solubility of PLA and PEG in chloroform is too different. Swaminathan et al. [17] studied the physical properties of PLA-PEG membranes $(L=35 \pm 5 \mu \mathrm{m})$ prepared by spin solvent-casting using PEG samples with different molecular weight. Membranes were found to be semiporous, the surface pore size depending on the PEG molecular weight. These findings suggested that the PLA:PEG mixtures used in this work are not the most appropriated for the fabrication of perforated PLA nanomembranes since the dependence on PEG characteristics is too large.

\section{PLA-PVA nanomembranes}

In order to overcome the limitations detected in PLA-PEG nanomembranes, PEG was replaced by PVA in the spin-coated mixtures. Both PLA and PVA are soluble in HFIP. This solvent exhibits a low evaporation temperature $\left(58.2{ }^{\circ} \mathrm{C}\right)$ and, therefore, a high evaporation rate is expected during the spin-coating process. However, it should be remarked that the sacrificial layer used to transform the supported PLA-PVA ultra-thin films into the PLA FsNM was also made of PVA. Therefore, the sacrificial layer may be significantly altered by the dropping of the HFIP solution mixture onto its surface, 
affecting the formation of the PLA-PVA film. In order to evaluate the impact of such effect two complementary experiments were performed.

First, a PLA solution $(10 \mathrm{mg} / \mathrm{mL})$ in HFIP was dropped onto a PVA sacrificial layer and spin-coated at $7000 \mathrm{rpm}$ for $1 \mathrm{~min}$. The resulting nanomembrane was characterized by AFM before and after being treated with water to eliminate the sacrificial layer (Figures $1 \mathrm{~b}$ and $1 \mathrm{c}$, respectively). It is worth noting that both the supported film and the FsNM exhibit a porous topography, even though the depth of such pores is smaller than the membrane thickness (Figure S2). These results clearly indicate the HFIP affects the PVA sacrificial layer during the spin-coating process. In spite of this, the apparition of nanofeatures (i.e. pores) in the PLA nanomembrane should be considered as good result within the context of this work.

In the second experiment, a droplet of HFIP was deposited onto the PVA sacrificial layer $(L=342 \pm 4 \mathrm{~nm}$ and $\mathrm{Rq}=0.3 \pm 0.1 \mathrm{~nm})$ and spin-coated at $7000 \mathrm{rpm}$ for $1 \mathrm{~min}$. After this, the thickness was not affected by the solvent $(L=344 \pm 48 \mathrm{~nm})$ while the roughness increased considerably $(\mathrm{Rq}=0.5 \pm 0.1 \mathrm{~nm})$. This variation, which is fully consistent with the apparition of nanofeatures in the previous experiment (Figures 1b and 1c), suggests that the instabilities created by the addition of a PLA:PVA solution in HFIP onto the PVA sacrificial layer could facilitate the fabrication of perforated PLA FsNMs.

PLA-PVA nanombrembranes were fabricated using 80:20 and 90:10 PLA:PVA mixtures in HFIP using the process schematically depicted in Figure 2. AFM topographic and phase images are displayed in Figure $3 a$ and $3 b$, respectively. As it can be seen, nanofeatures obtained for both compositions are apparently compatible with the formation of PVA nanophases crossing the thickness of the whole PLA nanomembrane (Table 1). However, comparison between the 80:20 and 90:10 PLA-PVA films reveals important differences related with the frequency and dimensional uniformity of PVA 
nanophases. Thus, 80:20 nanomembranes are not uniform, the dimensions and frequency of the nanophases depending on the examined region. In contrast, 90:10 films exhibit a relatively homogeneous distribution of PVA nanophases of similar dimensions. Such differences remained in the corresponding PLA FsNMs produced by selective water etching (Figure 3c and 3d). A Photograph displaying both a supported 90:10 PLA-PVA ultra-thin film and a nanoperforated 90:10 PLA FsNM floating in water is presented in Figure S3.

On the other hand, AFM images indicate that the pores obtained for the two compositions penetrate the entire thickness of the film, giving rise to perforations. Furthermore, SEM micrographs corroborate that perforations are irregular in terms of both distribution and dimensions in the FsNMs derived from the 80:20 PLA:PVA composition. Accordingly, regions with many small nanopores (Figure 3c, left) coexist in the same sample with regions exhibiting relatively large and infrequent pores (Figure 3c, right). In contrast, the 90:10 composition results in FsNMs with regular distribution of pores (Figure 3d).

\section{Characterization of perforated free-standing nanomembranes}

In order to enhance control over the distribution and diameter of nanoperforations obtained in FsNMs derived from 90:10 PLA-PVA nanomembranes, the mixing process of the two polymer solutions in HFIP was improved by applying vigorous stirring. More specifically, the 90:10 PLA:PVA mixture was stirred three times at $1000 \mathrm{rpm}$ for 30 second each one using a Vortex-type mixer. Hereafter, the resulting PVA-PLA nanomembranes as well as the corresponding PLA FsNMs have been denoted PLAPVA(stir) and PLA(stir) to differentiate them from those obtained without vigorous stirring. Figures 4 displays illustrative AFM images of PLA-PVA(stir) ultrathin films 
and PLA(stir) FsNMs, respectively. The main difference between the supported 90:10 PLA-PVA and PLA-PVA(stir) nanomembranes (Figures $3 \mathrm{~b}$ and $4 \mathrm{a}$, respectively) refers to the dimensions of the segregated nanophases, which are significantly smaller for the latter than for the former. After selectively solvent etching, the resulting nanoperforations (Figure 4b) exhibit not only a diameter reduction but also higher dimensional homogeneity.

SEM micrographs provided in Figure 5 display nanometric details on nanoperforated PLA and PLA(stir) FsNMs derived from 90:10 mixtures. Figure 5a illustrates the nanopores typically found in perforated 90:10 PLA, while submicrometric perforations rarely found in such membranes are shown in Figure $5 \mathrm{~b}$. The distribution of homogeneous nanopores in 90:10 PLA(stir) is evidenced in Figures 5c and 5d (low and high magnification, respectively), while details of such pores are given in Figures $4 \mathrm{e}$ and 5f. Finally, Figure 5g evidences the presence of nanopores at the two sides of the same 90:10 PLA(stir) FsNM. This indirectly proves that nanopores penetrate the entire thickness of the film, transforming into nanoperforations.

Quantitative measures of the pores, thickness and roughness were performed for perforated FsNMs derived from 80:20 and 90:10 PLA:PVA mixtures. For this purpose, five representative SEM images were considered for of each kind of nanomembrane. The average diameter and area of the pores $(\phi$ and $A$ ), the surface coverage by the perforations $(S)$, roughness and the thickness of such FsNM are listed in Table 2. As it was stated above, perforations in 80:20 PLA, which are the thickest ones, are submicrometric $(\phi \approx 0.8 \pm 0.5 \mu \mathrm{m}$ ), representing around $34 \%$ of the nanomembrane surface. However, inspection of the results obtained for 90:10 PLA indicates that the size of the pores $(\phi=170 \pm 73 \mathrm{~nm})$, the area covered by them $(S=6 \%)$ and the thickness decrease with the context of PVA in the mixture. Moreover, such three parameters 
experiences a more drastic reduction when the 90:10 PLA:PVA mixture is submitted to vigorous stirring, this feature being particularly important for the diameter of nanopores $(\phi=65 \pm 32 \mathrm{~nm})$. These results are fully consistent with detailed AFM depth profiles and monomodal pore diameter distribution displayed in Figures S4 and S5, respectively, which provide complementary information about the shape and size of the pores.

\section{Influence of nanoperforations in the PLA wettability}

Wettability, which is related with the surface topography, affects the cellular response of materials [18], determination of the influence of the perforations introduced in PLA ultra-thin films being required. For this purpose, the contact angle of milliQ water, $N, N$-dimethylformamide (DMF) and ethylene glycol (EG) were determined for non-perforated PLA, 90:10 PLA and 90:10 PLA(stir) nanomembranes supported on glass slips. Results, which are displayed in Figure 6a, indicate that nanoperforations do not provoke significant changes in the contact angle, independently of the solvent polarity. More specifically, the response of the three materials to water was very similar, indicating that the PLA hydrophilicity (i.e. contact angle $<90^{\circ}$ ) remains practically unaltered after creating nanopores crossing the entire membrane thickness.

Surface energies are related with contact angle measurements through the Young's equation [19. In this work, surface energies have been modelled using the Owen-WendtKaelble [20,21] (OWK) and Equation-of-State [22] (EOS) relationships, results being displayed in Figure 6b. As it can be seen, results provided by the two models are fully consistent. Thus, although the surface energies of non-perforated and perforated films do not exhibit important difference, those of latter are slightly smaller than that of the former for both OWK and EOS models. The fact that the surface energies of nanoperforated PLA are intermediate between those of non-perforated PLA and glass 
slip supports that nanopores cross the entire thickness of the nanomembranes. Moreover, this behaviour is consistent with the dimensions of the perforations, surface energies being slightly smaller for 90:10 PLA than for 90:10 PLA(stir).

\section{Influence of nanoperforations in cell viability}

Cell adhesion and proliferation on ultra-thin films depend on different factors, as for example the material used for their fabrication (i.e. chemical composition) and the surface topography, which in turn may altered through the introduction of nanofeatures. Thus, the dimensions and distribution of nanofeatures are expected to affect the behavior of nanomembranes as bioactive platforms. In this work the response of perforated 90:10 PLA and 90:10 PLA(stir) nanomembranes to cellular adhesion and proliferation have been compared with that of non-perforated PLA considering two cellular lines: Vero and MCDK, which are epithelial- like cells. It should be emphasized that such three PLA-based systems present identical chemical composition enabling us to obtain direct evidences of the influence of both the diameter and distribution of nanopores on cell growth. Quantitative results of cellular adhesion assays are displayed in Figure 7a. Results indicate that nanoperforations of $\phi \approx 170 \mathrm{~nm}$ promote cell adhesion with respect to the absence of perforations and, specially, nanoperforations of $\phi \approx 65 \mathrm{~nm}$. Thus, relative cell viability grows as follows: 90:10 PLA(stir) < non-perforated PLA < 90:10 PLA, differences being light more marked for MCDK cells than for Vero cells.

Cell proliferation assays were performed by evaluating the cellular activity on the three examined substrates after seven days of culture. Results, which are displayed in Figure $7 \mathrm{~b}$, show an appreciable increment in the cellular colonization for the two perforated nanomembranes. Evaluation of this increment with respect to the viability of adhered cells indicates that the preference of Vero and MCDK cells is higher for 90:10 
PLA(stir) than for non-perforated PLA, evidencing that perforations promote the interaction between the polymeric matrix and the cells once these have been adhered. However, the most remarkable result refers to perforated 90:10 PLA FsNMs, which exhibit cell viabilities $\sim 2.6$ and $\sim 2.2$ times higher than those of non-perforated PLA and perforated 90:10 PLA(stir), respectively. This marked preference has been attributed to the similarity between the diameters of nanoperforations in 90:10 PLA and the filopodia filaments in cells.

Filopodia are thin, actin-rich structures protruding from the lamellipodial actin network [23,24]. These elements, which are finger-like, sense the extracellular environment at their tips using cell surface receptors [25], playing an important role in cell migration. Thus, contact with an external target promotes the coupling of membrane-bound proteins to the backward flow of actin, which in turn produces the pulling forces needed for cell migration. This is essential for processes involving proliferation, as for example wound healing [26]. Contact differences between substrates influences the response of protruding filopodia. Interestingly, filopodia are 100-200 $\mathrm{nm}$ in diameter [23] while the diameter of the nanoperforations in 90:10 PLA is $170 \pm 73 \mathrm{~nm}$. Accordingly, nanoperforations in 90:10 PLA FsNMs promote cell migration processes, simultaneously favouring cell proliferation.

Figure 8 displays micrographs recorded by optical microscopy and SEM of Vero cells adhered onto 90:10 PLA and 90:10 PLA(stir) perforated nanomembranes. As it can be seen, cells adhered onto 90:10 PLA exhibit a radial heterogeneous spreading with respect to the center of the film (Figure 8a). This particular distribution, which is supported by the elongated morphology of the cells, clearly evidences the interaction between cells and 90:10 PLA nanofeatures. In contrast, cells adhered onto 90:10 PLA(stir) presents a random distribution in which regions without cells coexist with 
areas having a large accumulation of cells (Figure 8b). Furthermore, the morphology of the cells is less elongated than that of cells adhered onto 90:10 PLA. Finally, cells adhered onto non-perforated PLA follow a distribution similar to that described for 90:10 PLA(stir), where cells present a round-like morphology (Figure S6). On the other hand, observation of the micrographs displayed in Figure 7 evidences that ultra-thin films frequently exhibit micrometric striation flaws. These defects have been attributed to unfavorable capillary forces provoked by changes in surface tension during solvent evaporation [12,27]. This phenomenon, which is called "Marangoni instability", has been largely appreciated in ultra-thin films prepared by spin-coating. In this particular study, these tiny thickness undulations have very limited influence on the formation of the perforations as well as on cell adhesion and proliferation results.

The overall of these observations are fully consistent with our previous interpretation: cell elements interact intensely with nanofeatures of membranes with perforations of $\phi \approx 170 \mathrm{~nm}$ while these interactions become weaker when nanoperforations are too small and, especially, when they are non-existent.

\section{CONCLUSIONS}

In summary, nanoperforated PLA FsNMs of thickness 120-130 nm have been successfully obtained using spin-coating combined with phase segregation processes in immiscible 90:10 PLA:PVA mixtures and the subsequent removal of PVA domains via selective solvent etching. The diameter of the nanopores, which cross the entire nanomembrane thickness, has been controlled through the conditions applied to the preparation of the PLA:PVA mixture. Thus, the dimensional scale of the phase separation domain has been found to decrease when the mixing process is performed under vigorous stirring conditions. This procedure led to perforated PLA FsNM with 
nanopores of $\phi=170 \pm 73 \mathrm{~nm}$ (prepared without stirring) and $\phi=65 \pm 32 \mathrm{~nm}$ (prepared under vigorous stirring). The former diameter fits very well to the diameter of protruding finger-like filopodia $(100-200 \mathrm{~nm})$, which play an important role in cell migration processes. Cell proliferation assays considering non-perforated and perforated PLA nanomembranes as bioactive platforms indicate that ultra-thin films with nanopores of $\phi=170 \pm 73 \mathrm{~nm}$ exhibit cell viabilities that are $\sim 2.2$ and $\sim 2.6$ times higher than those with pores of $\phi=65 \pm 32 \mathrm{~nm}$ and non-perforated PLA, respectively. It is clear that this increase is enhanced by the better adhesion on such surfaces. Thus, nanopores of $\phi \approx 170 \mathrm{~nm}$ promote the substrate colonization through intense interactions with cell filopodia, as clearly demonstrate changes on both cell morphology and cell spreading pattern. Hence, nanoperforated FsNMs could be a great promise as novel bioactive platforms and would certainly improve the performance of current soft regenerative substrates. We are also planning to use nanoperforations to carry antibiotics and growth factors to aid the whole tissue regeneration process more effectively.

\section{ACKNOWLEDGEMENTS}

Authors are in debt to supports from MINECO and FEDER (MAT2012-34498).

\section{REFERENCES}

(1) H. Watanabe, E. Muto, T. Ohzono and T. Kunitake, J. Mater. Chem., 2009, 19, 2425-2431.

(2) K. D. Sattler, Handbook of Nanophysics, Functional Nanomaterials, CRC Press, Taylor and Francis Group, BocaRaton, FL, 2011.

(3) M. M. Pérez-Madrigal, E. Armelin, J. Puiggalí and C. Alemán, J. Mater. Chem. $B, 2015, \mathbf{3}, 5904-5932$. 
(4) Y. Okamura, K. Kabata, M. Kinoshita, H. Miyazaki, A. Saito, T. Fujie, S. Ohtsubo, D. Saitoh and S. Takeoka, Adv. Mater., 2013, 25, 545-551.

(5) T. Fujie, L. Ricotti, A. Desii, A. Menciassi, P. Dario and V. Mattoli, Langmuir, 2011, 27, 13173-13182.

(6) M. M. Pérez-Madrigal, E. Armelin, L. J. del Valle, F. Estrany and C. Alemán, Polym. Chem., 2012, 3, 979-991.

(7) M. M. Pérez-Madrigal, M. I. Giannotti, G. Oncins, L. Franco, E. Armelin, J. Puiggalí, F. Sanz, L. J. del Valle and C. Alemán, Polym. Chem., 2013, 4, 568583.

(8) Y. Okamura, K. Kabata, M. Kinoshita, D. Saitoh and S. Takeoka, Adv. Mater., 2009, 21, 4388-4392.

(9) H. Miyazaki, M. Kinoshita, A. Saito, T. Fujie, K. Kabata, E. Hara, S. Ono, S. Takeoka and D. Saitoh, Wound Repair Regen., 2012, 20, 573-579.

(10) V. Pensabene, S. Taccola, L. Ricotti, G. Ciofani, A. Menciassi, F. Perut, M. Salermo, P. Dario and N. Baldini, Acta Biomater., 2011, 7, 2883-2891.

(11) S. Walheim, M. Böltau, J. Mlynek, G. Krausch and U. Steiner, Macromolecules, 1997, 30, 4995-5003.

(12) H. Zhang and S. Takeoka, Macromolecules, 2012, 45, 4315-4321.

(13) R. Vendamme, A. Onoue, A. Nakao and T. Kunitake, Nat. Mater., 2006, 5, 494501.

(14) E. Armelin, A. L. Gomes, M. M. Pérez-Madrigal, J. Puiggalí, L. Franco, L. J. del Valle, A. Rodríguez-Galán, J. S. C. Campos, N. Ferrer-Anglada and C. Alemán, J. Mater. Chem., 2012, 22, 585-594.

(15) T. Mosmann, J. Immunol. Methods, 1983, 65, 55-63.

(16) S. Y. Heriot and R. A. L. Jones, Nat. Mater., 2005, 4, 782-786. 
(17) V. Swaminathan, R. Tchao and S. Jonnalagadda, J. Bimater. Sci. Polym. Ed., 2007, 18, 1321-1323.

(18) M. M. Pérez-Madrigal, M. I. Giannotti, L. J. del Valle, L. Franco, E. Armelin, J. Puiggalí, F. Sanz and C. Alemán, C. ACS Appl. Mater. Interfaces., 2014, 6, 97199732.

(19) P. J. Molino, M. J. Higgins, P. C. Innis, R. M. I. Kapsa and G. G. Wallace, Langmuir, 2012, 30, 2178-2187.

(20) A. W. Adamson, Physical chemistry of surfaces, 5th ed, John Wiley: New York, 1990.

(21) N. T. Correia, J. M. Ramos, B. Saramago and J. Calado, J. Colloid Interface Sci., 1997, 189, 361-369.

(22) D. K. Owens and R. C. Wendt, J. Appl. Polym. Sci., 1969, 13, 1741-1747.

(23) P. K. Mattila and P. Lappalainen, P. Nat. Rev. Mol. Cell Biol., 2008, 9, 446-454.

(24) X. B. Yuan, M. Jin, X. Xu, Y. Q. Song, C. P. Wu, M. M. Poo and S. Duan, Nat. Cell Biol., 2003, 5, 38-45.

(25) M. B. Steketee and K. W. Tosney, J. Neurosci., 2002, 22, 8071-8083.

(26) P. Lamoureux, R. E. Buxbaum and S. R. Heidemann, Nature, 1989, 340, 159162.

(27) D. P. Birnie, J. Mater. Res., 2001, 16, 1145-1154 
Table 1. Thickness $(L)$ and RMS roughness $(\mathrm{Rq})$ of PLA, PLA-PEG and PLA-PVA ultra-thin films prepared in this work. Observed nanofeatures are also indicated.

\begin{tabular}{|c|c|c|c|}
\hline & $L(\mathbf{n m})$ & $\mathbf{R q}(\mathbf{n m})$ & Nanofeatures \\
\hline PLA (chloroform) ${ }^{a}$ & $238 \pm 43$ & $1.1 \pm 0.2$ & - \\
\hline PLA-PEG (50:50) ${ }^{b}$ & $45 \pm 2$ & $4.0 \pm 1.0$ & - \\
\hline PLA-PEG $(60: 40)^{\mathrm{b}}$ & $48 \pm 2$ & $1.8 \pm 0.1$ & Shallow surface cavities \\
\hline PLA-PEG $(80: 20)^{b}$ & $48 \pm 2$ & $4.6 \pm 0.3$ & - \\
\hline PLA-PEG (90:10) ${ }^{b}$ & $50 \pm 4$ & $6.0 \pm 1.3$ & - \\
\hline PLA $(\text { HFIP })^{c}$ & $213 \pm 20$ & $2.8 \pm 0.3$ & - \\
\hline PLA-PVA $(80: 20)^{\mathrm{d}}$ & $74 \pm 3$ & $20.4 \pm 6.2$ & Nanophases penetrating the entire film \\
\hline PLA-PVA $(90: 10)^{d}$ & $62 \pm 8$ & $6.7 \pm 2.3$ & Nanophases penetrating the entire film \\
\hline $\begin{array}{l}\text { PLA-PVA (90:10) (with } \\
\text { vigorous stirring) }^{d}\end{array}$ & $96 \pm 2$ & $3.3 \pm 1.2$ & Nanophases penetrating the entire film \\
\hline \multicolumn{4}{|c|}{$\begin{array}{l}{ }^{a} \text { PLA ultra-thin film prepared by spin-coating a } 10 \mathrm{mg} / \mathrm{mL} \text { polymer }(10 \mathrm{mg} / \mathrm{mL}) \\
\text { chloroform solution. }{ }^{b} \text { PLA-PEG ultra-thin films prepared using by spin-coating PLA } \\
(10 \mathrm{mg} / \mathrm{mL}): \text { PEG }(10 \mathrm{mg} / \mathrm{mL}) \text { mixtures in chloroform. }{ }^{c} \text { PLA ultra-thin film prepared } \\
\text { by spin-coating a } 10 \mathrm{mg} / \mathrm{mL} \text { polymer }(10 \mathrm{mg} / \mathrm{mL}) \mathrm{HFIP} \text { solution. }{ }^{\mathrm{d}} \text { PLA-PVA ultra-thin } \\
\text { films prepared using by spin-coating PLA }(10 \mathrm{mg} / \mathrm{mL}): \text { PVA }(10 \mathrm{mg} / \mathrm{mL}) \text { mixtures in } \\
\text { HFIP. }\end{array}$} \\
\hline
\end{tabular}


Table 2. Average diameter $(\phi)$ and area $(A)$ of the pores, surface coverage by pores $(S)$, RMS roughness $(\mathrm{Rq})$ and thickness $(L)$ of perforated PLA FsNMs derived from 80:20 and 90:10 PLA:PVA mixtures.

\begin{tabular}{cccccc}
\hline FsNM & $\phi(\mathbf{n m})$ & $\boldsymbol{A}\left(\mathbf{n m}^{2}\right)$ & $\boldsymbol{S}(\boldsymbol{\%})$ & $\boldsymbol{R q}(\mathbf{n m})$ & $\boldsymbol{L}(\mathbf{n m})$ \\
\hline PLA & - & - & - & $1.1 \pm 0.2$ & $116 \pm 14$ \\
80:20 PLA & $841 \pm 546$ & $555493 \pm 242886$ & $34 \%$ & $186.9 \pm 25.6$ & $170 \pm 14$ \\
90:10 PLA & $170 \pm 73$ & $22713 \pm 4225$ & $6 \%$ & $93.2 \pm 31.2$ & $127 \pm 10$ \\
90:10 PLA(stir) & $65 \pm 32$ & $3291 \pm 795$ & $1 \%$ & $108.9 \pm 5.7$ & $117 \pm 16$ \\
\hline
\end{tabular}




\section{CAPTIONS TO FIGURES}

Figure 1. (a) Three dimensional height (left) and phase (right) AFM images of PLA supported nanomembrane prepared using a $10 \mathrm{mg} / \mathrm{mL}$ polymer solution in chloroform and a spin-coater speed of $7000 \mathrm{rpm}$. AFM images of PLA (b) the supported nanomembrane and (c) the corresponding FsNM prepared using a $10 \mathrm{mg} / \mathrm{mL}$ polymer solution in HFIP and a spin-coater speed of $7000 \mathrm{rpm}$. The FsNM was obtained after removing the sacrificial layer of the supported nanomembrane. The AFM image windows are $10 \times 10 \mu \mathrm{m}^{2}$ in all cases.

Figure 2. Schematic representation of the procedure used to prepare perforated PLA nanomembranes from PLA-PVA ultra-thin films by the spin-coating technique.

Figure 3. Three dimensional height (left) and phase (right) AFM images of PLAPVA supported nanomembranes prepared using a (a) 80:20 and (b) 90:10 PVA:PLA mixture in HIP. Nanoperforated PLA FsNMs obtained via selective water etching using the (c) 80:20 and (d) 90:10 PLA-PVA supported nanomembranes: Three dimensional height (left) and phase (right) AFM images, and representative SEM micrographs (bottom). The AFM image windows are $10 \times 10 \mu \mathrm{m}^{2}$ in all cases.

Figure 4. Three dimensional height (left) and phase (right) AFM images of (a) PLAPVA supported nanomembranes prepared using a vigorously stirred 90:10 PVA:PLA mixture in HIP and (b) nanoporferated PLA FsNMs obtained via selected water etching using the supported nanomembranes displayed in (a). In all cases image windows are $10 \times 10 \mu \mathrm{m}^{2}$ (top) and $10 \times 10 \mu \mathrm{m}^{2}$ (bottom).

Figure 5. SEM micrographs of nanoperforated (a and b) 90:10 PLA and (c-g) 90:10 PLA(stir) FsNMs prepared using 90:10 PLA:PVA mixtures in HFIP. Details on a-f are described in the text. A folded 90:10 PLA(stir) FsNM showing the presence of 
nanoperforations at the two sides is displayed in $(\mathrm{g})$. This SEM image reflects that nanoperforations cross the thickness of the nanomembrane.

Figure 6. (a) Contact angle determined of milliQ water, $N, N$-dimethylformamide (DMF) and ethylene glycol (EG). (b) Surface energy calculated using Owen-WendKaelble (OWK) and Equation-of-Stat (EOS) models for non-perforated PLA, 90:10 PLA and 90:10 PLA(stir) FsNMs.

Figure 7. (a) Cellular adhesion and (b) cellular proliferation on the surface of nanoperforated 90:10 PLA and PLA(stir) membranes and non-perforated PLA membranes. Vero and MCDK cells were culture during $24 \mathrm{~h}$ (adhesion assay) and 7 days (proliferation assay). The experiments were performed using four samples for each substrate.

Figure 8. Optical microscopy (left) and SEM (right) images of Vero cells adhered onto perforated (a) 90:10 PLA and (b) 90:10 PLA(stir) nanomembranes. 
(a)

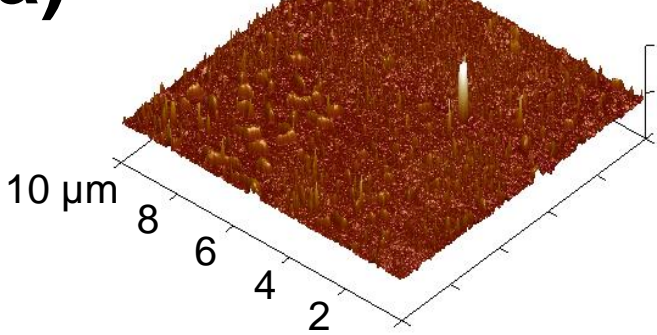

(b)

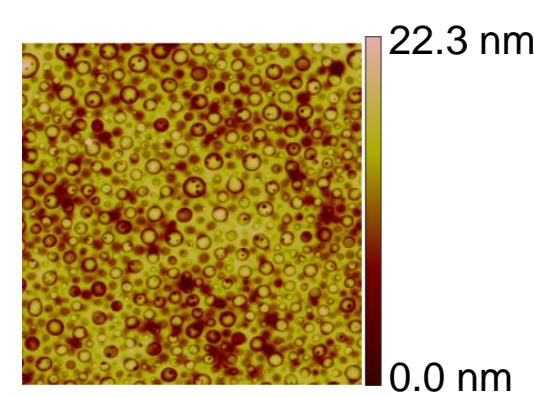

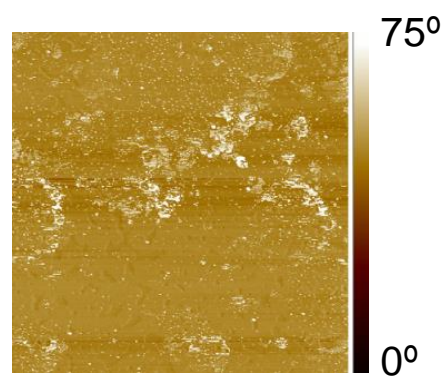

(c)

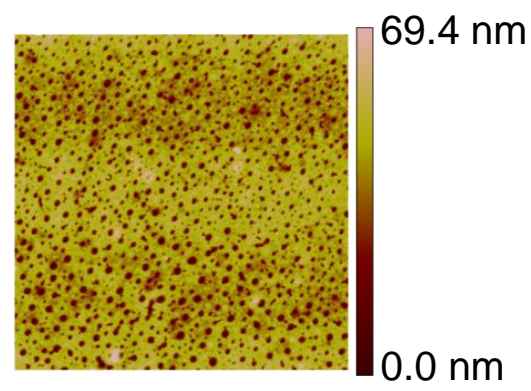

Figure 1 


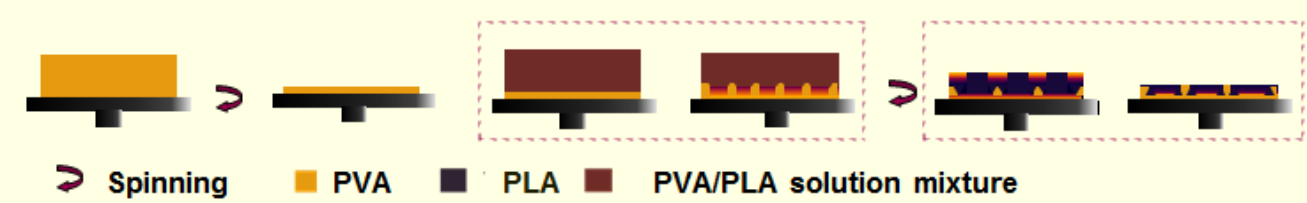

Figure 2 

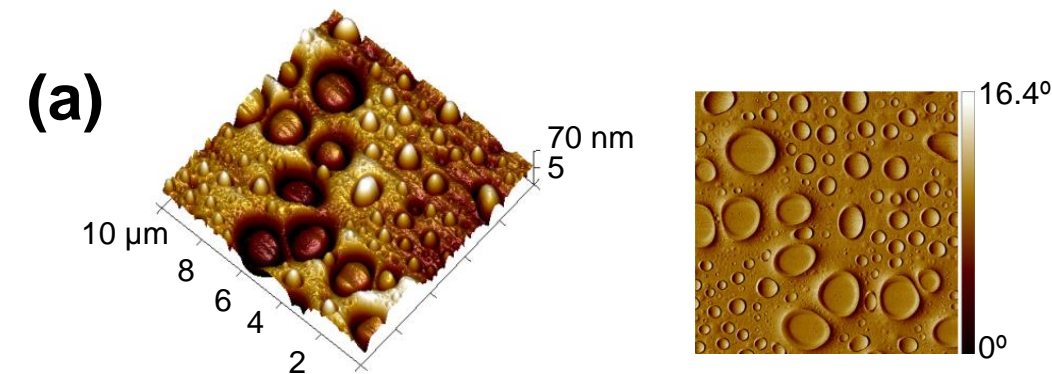

(b)
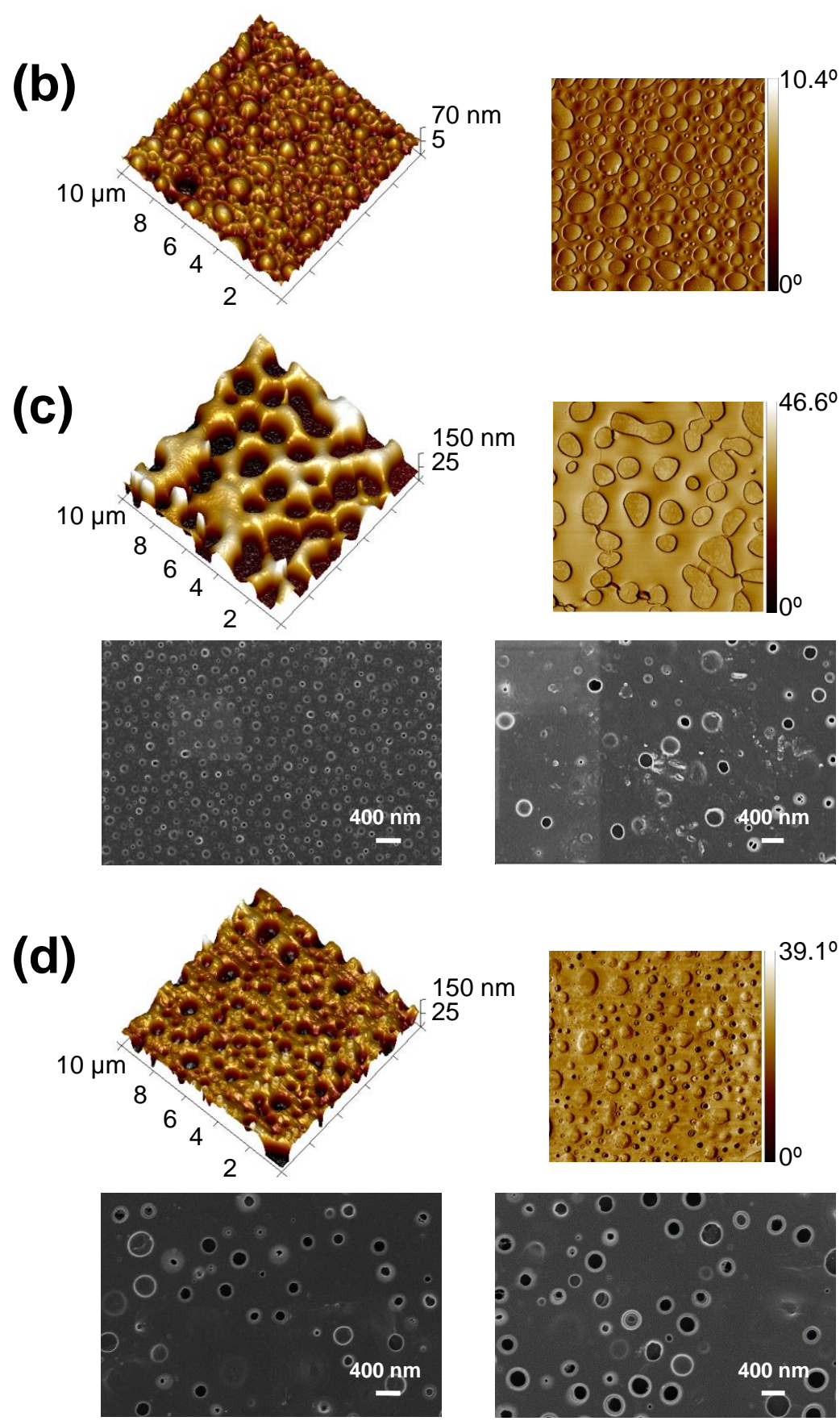

Figure 3 
(a)
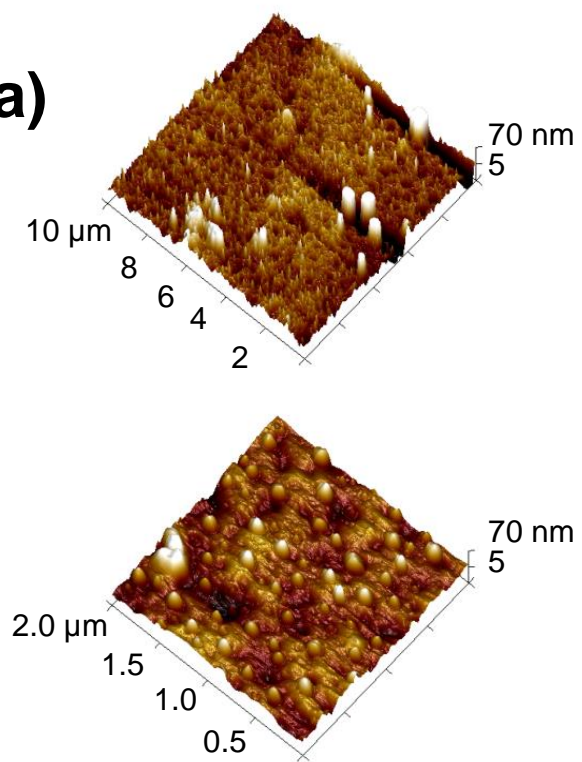

(b)
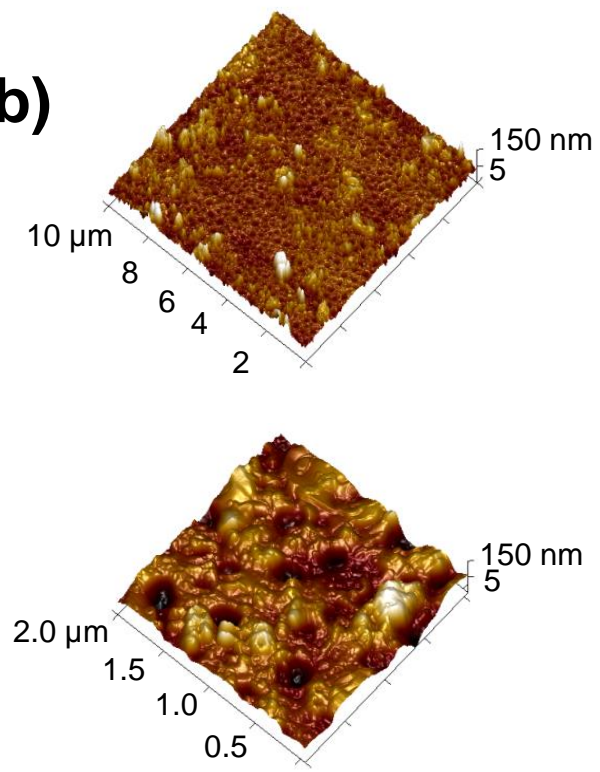
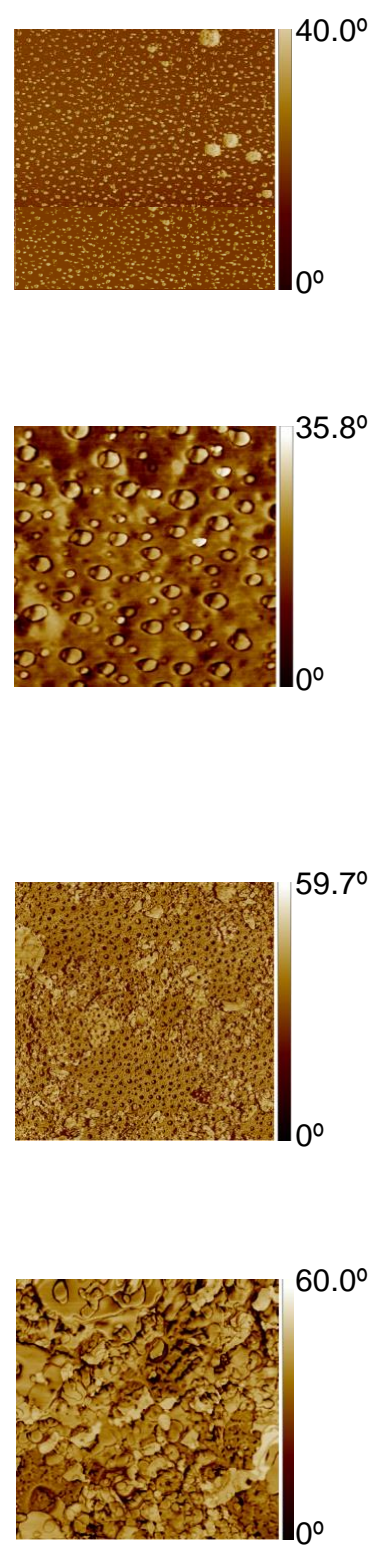

Figure 4 

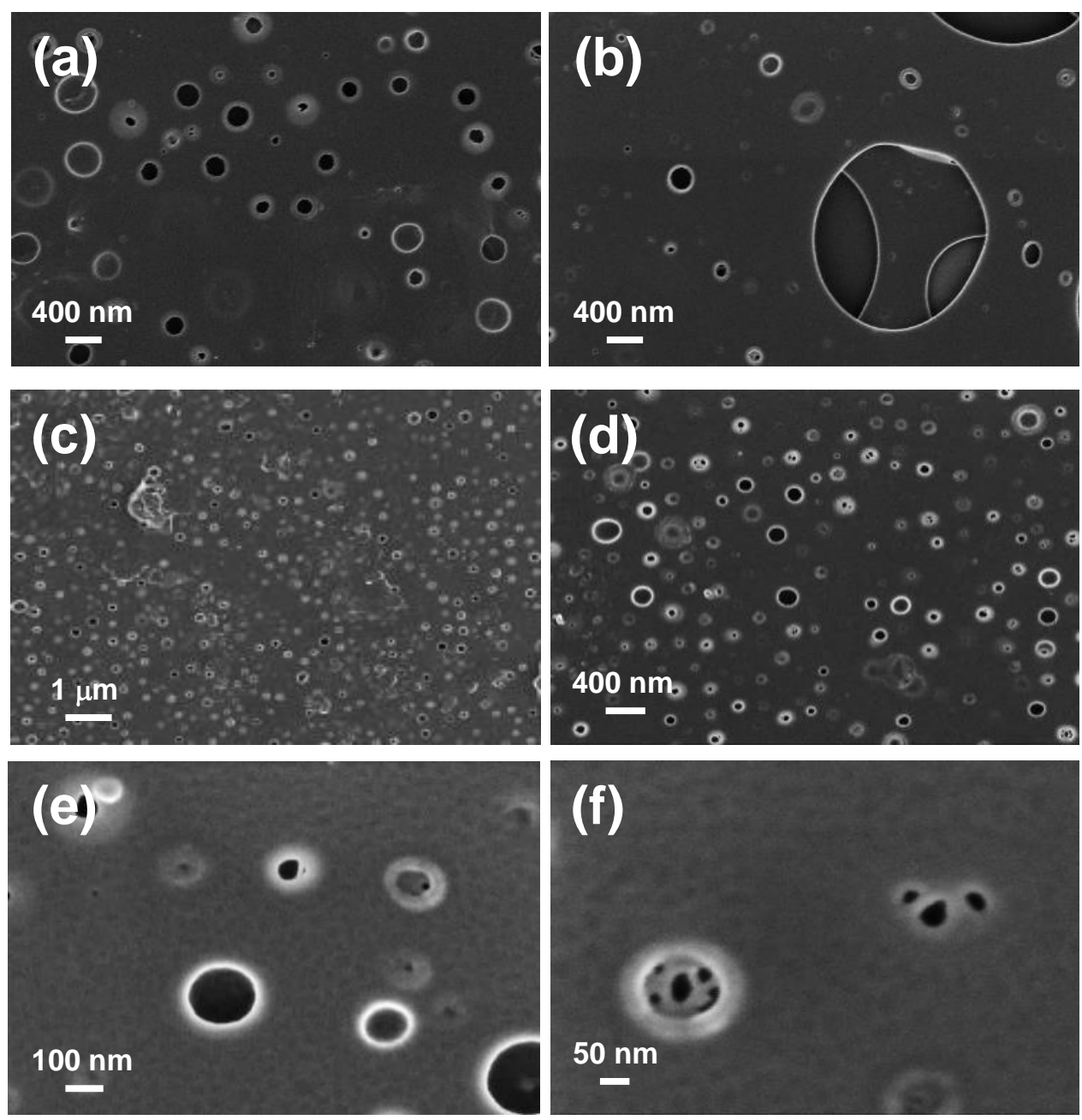

(f)

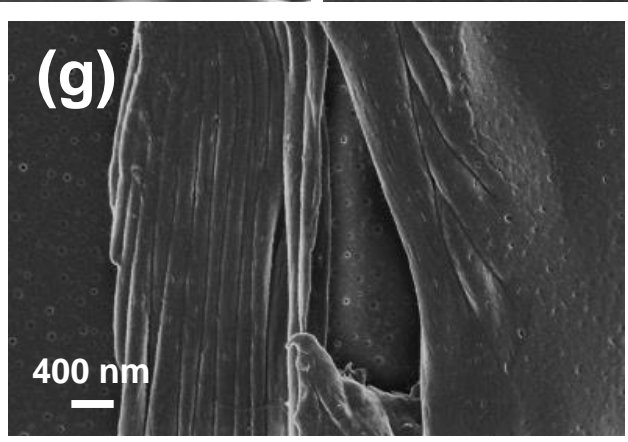

Figure 5 
(a)

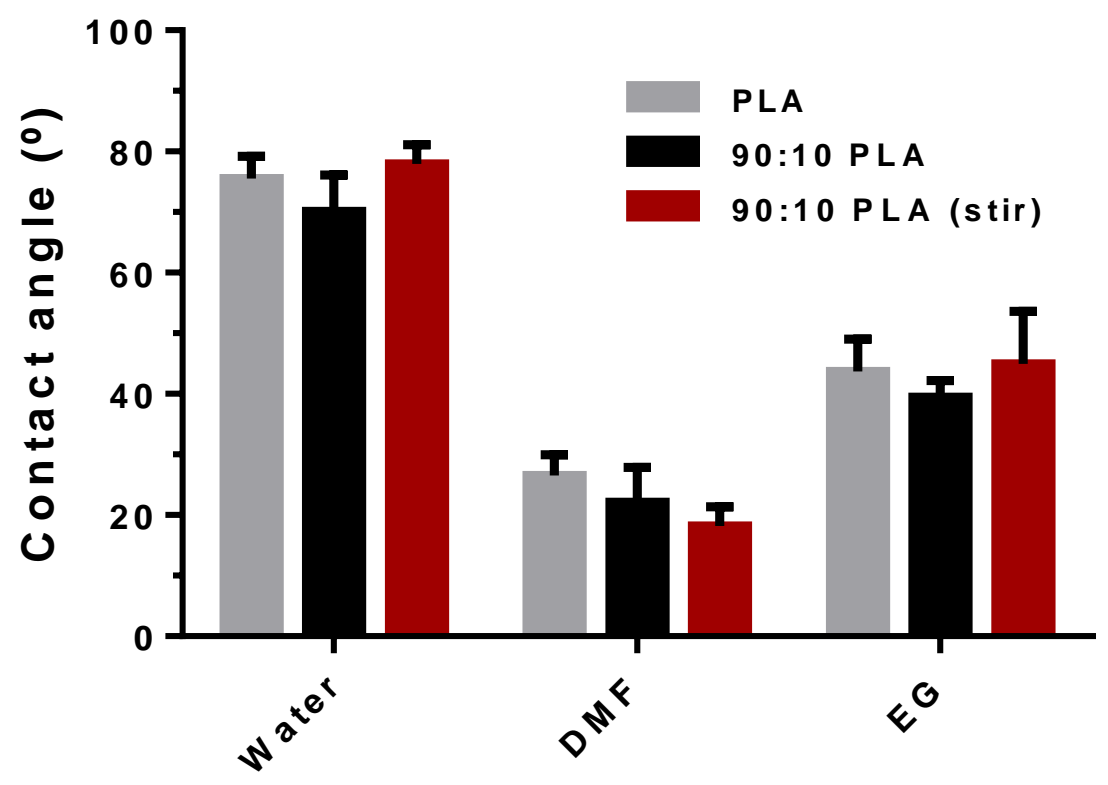

(b)

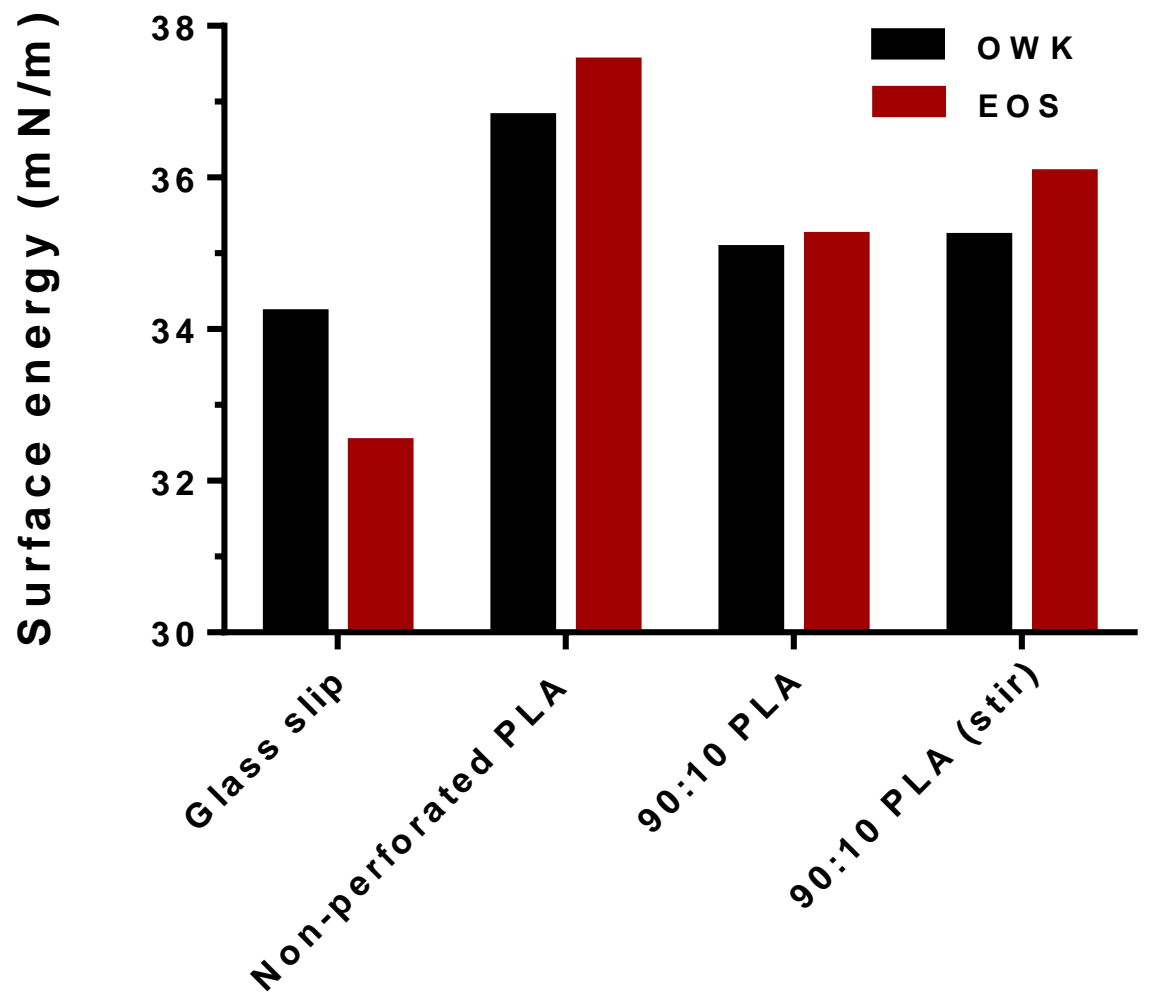

Figure 6 
(a)

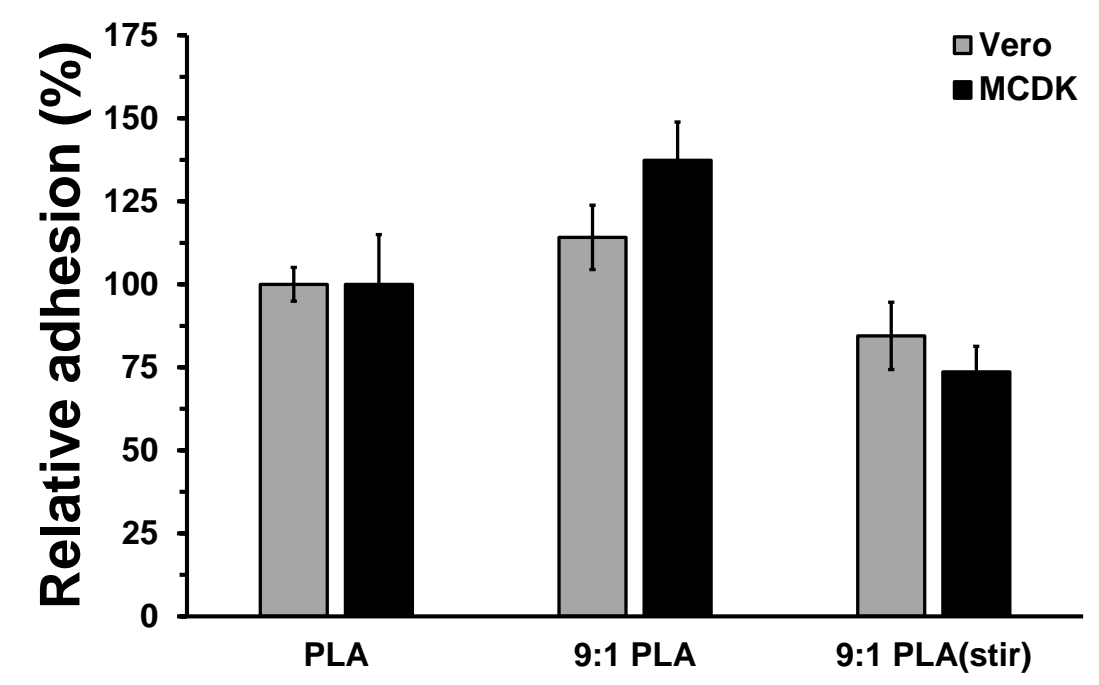

(b)

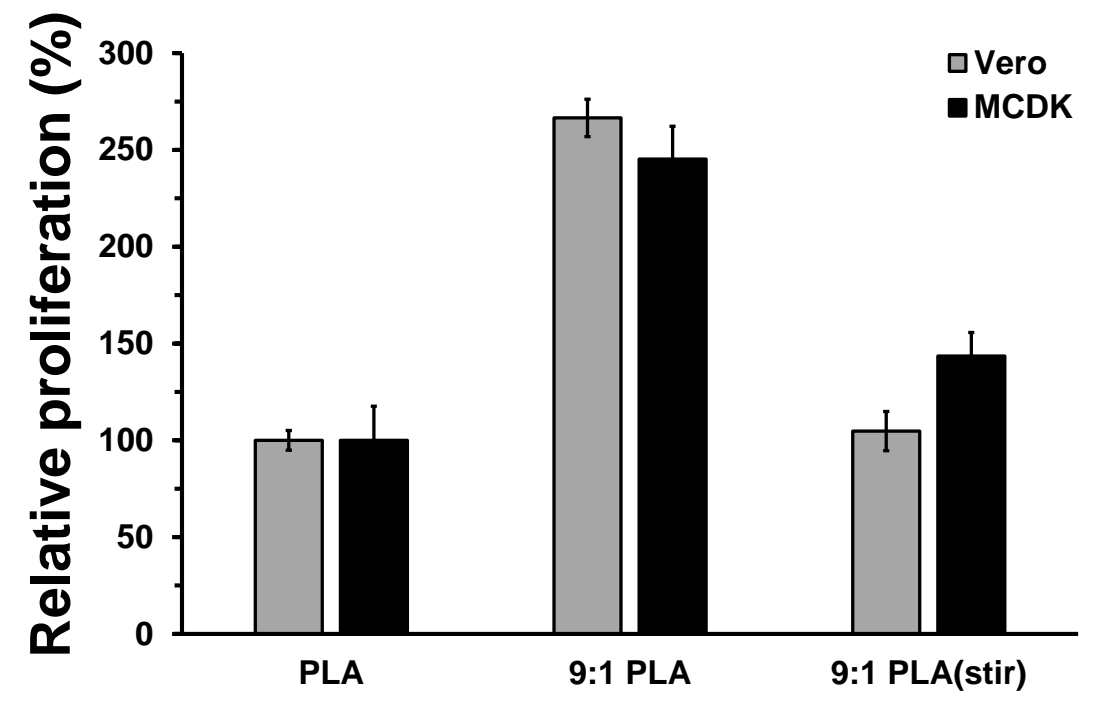

Figure 7 

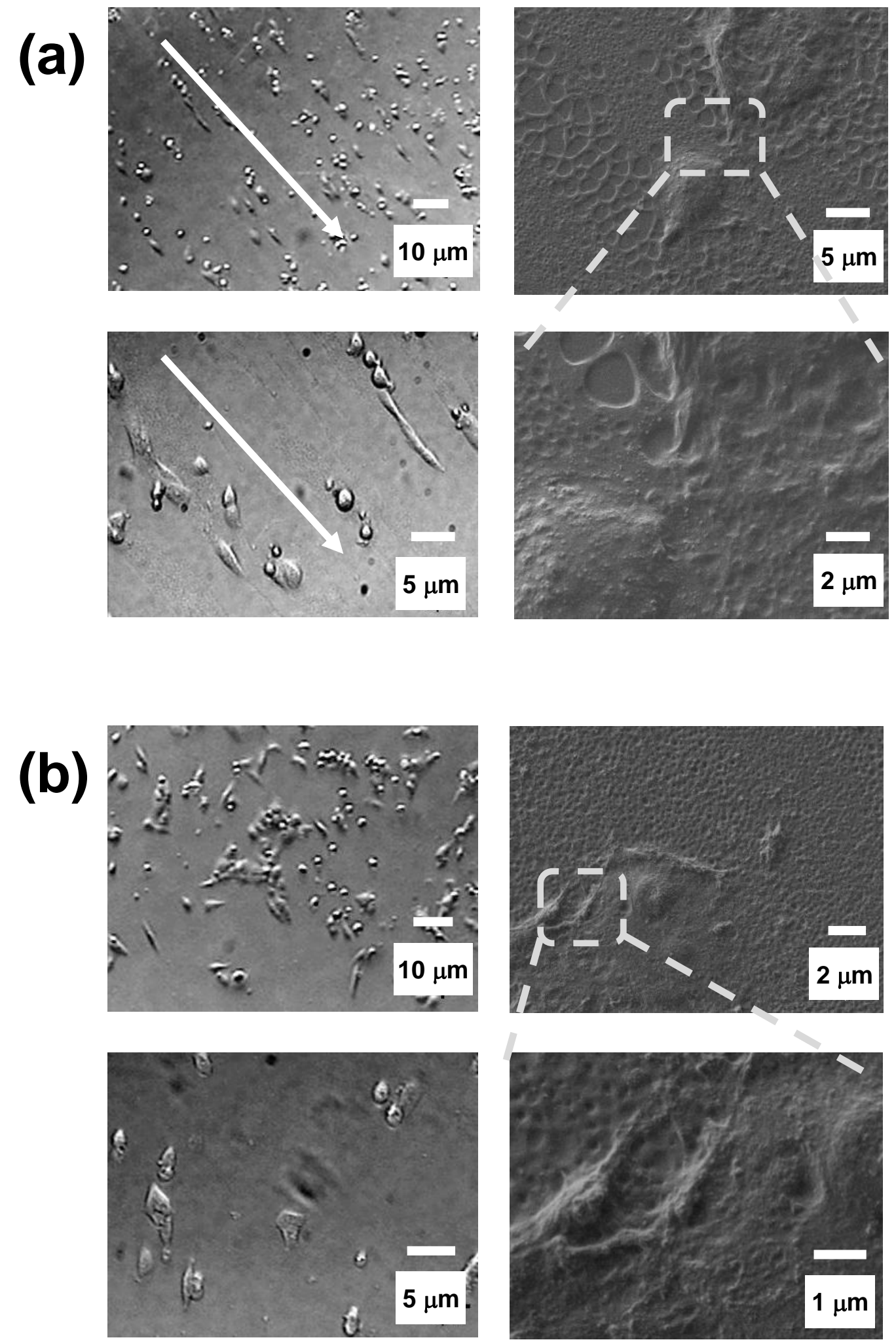

Figure 8 